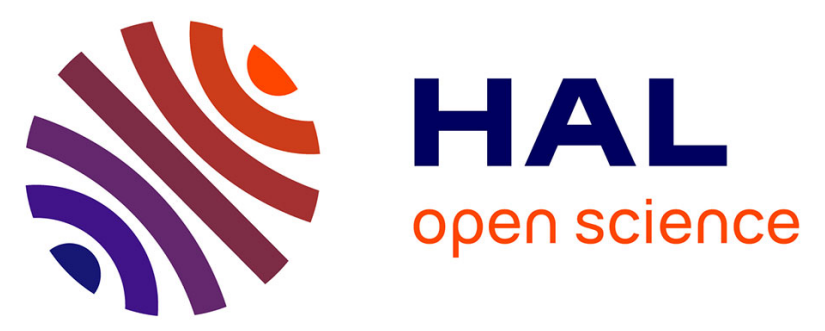

\title{
Three repeated sessions of transcranial random noise stimulation (tRNS) leads to long-term effects on reaction time in the Go/No Go task
}

Charlotte Brevet-Aeby, Marine Mondino, Emmanuel Poulet, Jérome Brunelin

\section{- To cite this version:}

Charlotte Brevet-Aeby, Marine Mondino, Emmanuel Poulet, Jérome Brunelin. Three repeated sessions of transcranial random noise stimulation (tRNS) leads to long-term effects on reaction time in the Go/No Go task. Neurophysiologie Clinique = Clinical Neurophysiology, 2019, 49, pp.27 - 32. 10.1016/j.neucli.2018.10.066 . hal-03486194

\author{
HAL Id: hal-03486194 \\ https://hal.science/hal-03486194
}

Submitted on 20 Dec 2021

HAL is a multi-disciplinary open access archive for the deposit and dissemination of scientific research documents, whether they are published or not. The documents may come from teaching and research institutions in France or abroad, or from public or private research centers.
L'archive ouverte pluridisciplinaire HAL, est destinée au dépôt et à la diffusion de documents scientifiques de niveau recherche, publiés ou non, émanant des établissements d'enseignement et de recherche français ou étrangers, des laboratoires publics ou privés.

\section{(c) (1) $\$$}

Distributed under a Creative Commons Attribution - NonCommerciall 4.0 International 


\section{Original article}

Three repeated sessions of transcranial random noise stimulation (tRNS) leads to long term effects on reaction time in the Go/No Go task.

Charlotte BREVET-AEBY 1,2,3, Marine MONDINO 1,2,4, Emmanuel POULET ${ }^{1,2,3,4}$, Jérome BRUNELIN 1,2,4

1 INSERM U1028; CNRS UMR5292; Lyon Neuroscience Research Center, IR2 Team, Lyon, F-69000, France;

${ }^{2}$ University Lyon 1, Villeurbanne, F-69000, France;

${ }^{3}$ Emergency Psychiatry, Edouard Herriot Hospital, Hospices Civils de Lyon, France

${ }^{4}$ Centre Hospitalier Le Vinatier, 69678 Bron, France;

\section{Correspondence}

\section{Charlotte BREVET-AEBY}

Centre Hospitalier Le Vinatier, Pôle Est - Bâtiment 416 - 1er étage,

BP 30039 - 95 boulevard Pinel

\section{BRON Cedex - FRANCE}

charlotte.brevet@ gmail.com / Phone Number: +33 (0) 437915565 


\section{Abstract}

Objective: We aimed to investigate whether the number of sessions delivered within one day influences the acute and long-term effects of transcranial random noise stimulation (tRNS) applied over the dorsolateral prefrontal cortex (DLPFC) on inhibitory control.

Methods: Thirty-six healthy participants were randomly assigned to receive either (i) 3 active (3A), (ii) 1 active and 2 sham (1A2S) or (iii) 3 sham (3S) tRNS sessions. The 3 tRNS were delivered consecutively in the same half-day separated by 30 min with the anode over the right and the cathode over the left DLPFC. The effects of tRNS on inhibitory control were assessed 5 times using a Go/No Go task: at baseline (T0), after one tRNS session (T1), after the 3 sessions (T3), 1 (D1) and 8 (D8) days after.

Results: As compared to sham, active tRNS did not acutely modulate accuracy in the Go/No Go task but decreased reaction times at Go trials. At D1, participants who received at least one active tRNS sessions (3A and $1 \mathrm{~A} 2 \mathrm{~S}$ groups) were faster than those who received 3 sessions of sham. At D8, only the participants who received 3 active tRNS sessions were faster to successfully respond to Go trials as compared to sham.

Discussion: Three active tRNS sessions induced longer effects on reaction times than a single session of active tRNS, compared to sham. These findings suggested that delivering repeated tRNS sessions on the same day induces more sustained effects than delivering a single session.

Keywords: dorsolateral prefrontal cortex; Go/No Go task; impulsive behavior; inhibitory control; noninvasive brain stimulation; transcranial random noise stimulation 


\section{Introduction}

Inhibitory control, a key component of impulsivity, is the capacity to inhibit inappropriate responses or actions by suppressing irrelevant information [1]. This process plays a major role in everyday activities and is known to be affected in several psychiatric conditions, such as obsessive compulsive disorder, schizophrenia, attention-deficit hyperactivity disorder as well as suicidal behaviors [1,4].

Numerous neuroimaging studies have investigated brain correlates of inhibitory control. A consistent finding is that inhibitory control involved a mainly right lateralized network, including the inferior frontal gyrus, the anterior cingulate and the dorsolateral prefrontal cortex (DLPFC) as well as parietal and subcortical areas [e.g., $8,13,17,20]$. The involvement of the DLPFC in inhibitory control is also supported by studies showing that transcranial direct current stimulation (tDCS) applied over the right and/or the left DLPFC can transitorily modulate inhibitory control in healthy volunteers [5]. tDCS is a noninvasive technique known to modulate brain activity and connectivity in-vivo by applying a weak electrical current between two electrodes place over the scalp of the subject. Interestingly, tDCS has been used to study relationships between brain and functions. Regarding inhibitory control, Beeli et al. [3] showed that one session of tDCS applied with the cathode over the right DLPFC and the anode over the ipsilateral mastoid can increase the number of false alarms during a Go/No-go task as compared to sham and to anodal stimulation. Another study reported that tDCS applied with the cathode over the left DLPFC coupled with the anode over the right supraorbital region can significantly impair response inhibition in a Go/No Go task [19]. Thus, even if the electrode montage differed between studies, when taken together these studies highlight the crucial role of the DLPFC in inhibitory control and have raised interest in using tDCS over the DLPFC 
in patients with psychiatric conditions, in order to increase their inhibitory control performance. In this way, Boggio et al. [6] reported that tDCS applied with the anode over the left DLPFC and the cathode over the right supraorbital area can increase accuracy at a Go/No-Go task in patients with major depressive disorder. Soltaninejad et al. [21] have showed that tDCS with the cathode over the left DPLFC and the anode over the right supraorbital area can increase inhibition accuracy in adolescents with ADHD symptoms.

Thus, tDCS appears promising to modulate inhibitory control. However, little is known regarding the optimal stimulation parameters to apply in order to provide a therapeutic application with sustained beneficial effects. With that in mind, two augmentation strategies have been proposed: increasing the number of tDCS sessions and changing the shape of the current. The first strategy (i.e. increasing the number of sessions) is based on neurophysiological studies investigating the effects of tDCS applied over the motor cortex [15,18]. Namely, Monte-Silva et al. reported that delivering repeated sessions of tDCS in the same day increase the duration of the effect on motor excitability. Ho et al. [14] showed that applying a single tDCS session over consecutive days result in a cumulative effect on motor excitability. Interestingly, cumulative effects of tDCS were also observed on behavioral outcomes when tDCS was applied over the DLPFC [7]. However, some other studies found no additional effects of repeated sessions of tDCS [e.g., 22,25]. The second strategy (i.e. changing the shape of current) is based on studies comparing the neurophysiological effects of different shape of stimulation. Namely, some studies reported that transcranial Random Noise Stimulation (tRNS) can induce more intense effects on neural plasticity [16] and on behavioral outcomes than classical DC stimulation [11]. tRNS consists of delivering an oscillating current at random amplitude and 
frequencies and is suggested to increase cortical excitability, especially when using high frequencies [23]. Comparing two methods of tRNS, Ho and colleagues reported that tRNS with a DC offset is more likely to lead increases in motor cortex excitability than tRNS with no DC offset [15]. However, these two augmentation strategies (i.e., repeating the number of sessions and applying tRNS) have not been tested on inhibitory control.

Here, we aimed to investigate the acute and long-term effects of three 20-min consecutive repeated sessions of tRNS applied over the DLPFC on inhibitory control in healthy subjects. The 3 sessions of tRNS were delivered in the same day and separated by $30 \mathrm{~min}$. We hypothesized that three sessions of active tRNS would induce longer beneficial effects on inhibitory control as compared with a single session of active tRNS and with sham tRNS. To measure inhibitory control processes, we used a Go/No-Go task, a commonly used paradigm in which subjects are required to respond as quickly as possible to frequent go trials and refrain from responding on non-frequent no-go trials.

\section{Methods}

\section{Participants}

In a randomized double-blind 3-arm parallel study, 36 healthy participants (with no history of neurological or psychiatric condition, no psychotropic medication and no contraindication to tRNS) gave their written informed consent before receiving 3 consecutive tRNS sessions separated by 30 minutes (figure 1A): either (i) 3 sessions of active tRNS (3A group), (ii) 1 session of active tRNS followed by 2 sessions of sham tRNS (1A2S group) or (iii) 3 sessions of sham tRNS (3S group). Three participants were excluded from the analyses (loss of data from 1 participant; 2 
participants were outliers according to Dixon's test). The final analyzed sample consisted of 33 participants, 12 in the $3 \mathrm{~A}, 10$ in the $1 \mathrm{~A} 2 \mathrm{~S}$ and 11 in the $3 \mathrm{~S}$ group (mean age: 24.72 years +/- standard deviation: 4.32; 17 women and 16 men, two lefthanded). The study was approved by the local ethics committee (CPP Sud Est 6, AU1222) and registered in ClinicalTrials.gov database (NCT02717260). Impulsivity was assessed at baseline using the Barratt Impulsive Scale (BIS-10, Table 1).

\section{Inhibitory control assessment}

Participants performed a standardized verbal Go/No-Go task 5 times: 1) before (T0), 2) after the first tRNS session (T1), 3) after the third tRNS session (T3), 4) one day after (D1) and 5) 8 days after (D8) the three tRNS sessions._The Go/No-Go task was implemented in DMDX display software [12] and consisted of 60 trials (40 Go, 20 No-Go). Trials were presented with a different randomized order in each of the sessions to avoid learning effect. Participants were instructed to respond as fast and accurately as possible to the Go stimulus (the letter 'B') by pressing a button, while inhibiting their response to the No-Go stimulus ('E'). Each trial was presented on a 17' computer screen at $60 \mathrm{~cm}$ of the head and consisted of a fixation cross for $200 \mathrm{~ms}$ followed by a white screen for $200 \mathrm{~ms}$ and a letter stimulus for $1500 \mathrm{~ms}$ terminating as soon as the participants responded. (figure 1B)

To reflect accuracy, number of omission errors (incorrectly withholding a response on a go trial) and false alarms (incorrectly responding to a No-Go trial) were also measured. However, since none of the participants made omission errors, only false alarms were analyzed. For each participant, the mean reaction time for correct responses at Go trials was calculated. Trials with reaction times that differed from the 
individual mean by \pm 2 standard deviations were considered outliers and removed (< $5 \%$ of trials).

\section{Transcranial Random Noise Stimulation (tRNS)}

High-frequency oscillatory direct current tRNS $(100-500 \mathrm{~Hz})$ set at $2 \mathrm{~mA}$ intensity with a $1 \mathrm{~mA}$ offset was delivered for $20 \mathrm{~min}$ with a 30-second ramp up/ramp down periods between two sponge electrodes $(7 \times 5 \mathrm{~cm})$ using the StarStim device (NeuroElectrics, Barcelona, Spain). The anode was placed over the right DLPFC (F4 according to the 10/20 electrode placement EEG-system) and the cathode over the left DLPFC (F3). Sham tRNS consisted of the same ramp up/ramp down periods at the beginning of stimulation session but no actual stimulation was delivered during the rest of the stimulation period. Blinding integrity was assessed at T3 by asking participants to guess the nature of the received condition (3A, 1A2S or $3 \mathrm{~S}$ group).

\section{Statistical analysis}

Statistical analyses were performed using SPSS-22 (Statistical Package for the Social Sciences) with a significance level of $\mathrm{p}<0.05$ (two-tailed). Sociodemographic and clinical characteristics were compared between groups using one-factor ANOVAs for quantitative data and Fisher's exact tests for qualitative data. Changes in reaction times at Go trials (expressed as a ratio to baseline) were compared using a repeated measures ANOVA with group (3A, 3S, 1A2S) as a between-subjects factor and time (T0, T1, T3, D1, D8) as a within-subjects factor. In the case of significance, Fisher's Least Significant Difference (LSD) post-hoc tests were performed for between-group comparisons (12 comparisons). 


\section{Results}

\section{Participants}

There was no significant difference between groups regarding gender, age, educational level, and BIS-10 scores (see Table 1).

$* * * * * * * * * * * * * * * * * * * * * * * \mathrm{TABLE} 1 * * * * * * * * * * * * * * * * * * * * * * * * * *$

\section{Effects on accuracy (number of false alarms)}

Number of false alarms was very low at baseline (mean $=0.58+/-0.87)$ and $60 \%$ of the participants made no false alarms. The ANOVA on the number of false alarms revealed no significant main effect of Time $(F=1.59 ; p=0.18 ; \eta 2=0.05)$, Group $(F$ $=1.04 ; \mathrm{p}=0.37 ; \eta 2=0.06)$ and no significant interaction between Time and Group $(\mathrm{F}=0.486 ; \mathrm{p}=0.86 ; \eta 2=0.03)$.

\section{Effects on reaction times}

The ANOVA on changes in reaction times at Go trials revealed a significant main effect of Time $\left(F=10.8 ; p<0.001 ; \eta^{2}=0.26\right)$, Group $\left(F=4.5 ; p=0.019 ; \eta^{2}=0.23\right)$ and a significant interaction between Time and Group $\left(F=2.13 ; p=0.038 ; \eta^{2}=0.12\right.$, figure 1c). Between-group post-hoc tests revealed that the participants from the $3 \mathrm{~A}$ group responded significantly faster at Go trials than those from the $3 \mathrm{~S}$ group at $\mathrm{T} 1(\mathrm{p}=0.007), \mathrm{D} 1(\mathrm{p}=0.003)$ and $\mathrm{D} 8(\mathrm{p}=0.035)$ but not at $\mathrm{T} 3(\mathrm{p}=0.140)$.

The 1A2S group significantly differed from the $3 \mathrm{~S}$ group at T1 $(\mathrm{p}=0.046)$ and D1 $(\mathrm{p}$ $=0.028)$ but not at $\mathrm{T} 3$, where only a trend toward significance was observed $(\mathrm{p}=$ 0.053), and not at D8 $(\mathrm{p}=0.217)$. Interestingly, there was no significant difference 
between $3 \mathrm{~A}$ and $1 \mathrm{~A} 2 \mathrm{~S}$ at any times of measure $(\mathrm{T} 1, \mathrm{p}=0.488 ; \mathrm{T} 3, \mathrm{p}=0.567 ; \mathrm{D} 1, \mathrm{p}=$ 0.465; D8, $\mathrm{p}=0.392)$.

When corrected for multiple comparisons using Bonferroni correction, only the $3 \mathrm{~A}$ group remained significantly different from the $3 \mathrm{~S}$ group at $\mathrm{T} 1\left(\mathrm{p}_{\text {corr }}=0.021\right)$ and $\mathrm{D} 1$ $\left(\mathrm{p}_{\text {corr }}=0.010\right)$.

$* * * * * * * * * * * * * * * * * * * * * * * \mathrm{FIGURE} 1 * * * * * * * * * * * * * * * * * * * * * * * * * *$

Safety and integrity of blinding

tRNS was well tolerated, none of the subjects reporting adverse effects during the follow-up period. All participants described a slight itching sensation under the electrodes during the first 30-second of stimulation. Regarding blinding, only 3 participants correctly guessed their stimulation condition 2 in the $1 \mathrm{~A} 2 \mathrm{~S}$ group and 1 in the $3 \mathrm{~A}$ group.

\section{Discussion}

In the present study, we applied tRNS over the DLPFC to investigate whether random oscillatory currents in this region affect inhibitory control processes in healthy subjects. Moreover, we investigated whether delivering three repeated sessions of active tRNS in the same day separated by 30 min would induce longer effects on inhibitory control as compared to a single session of active tRNS, and to sham tRNS. We failed to demonstrate any beneficial or deleterious acute or long-term effects of tRNS on accuracy at the Go/NoGo task, suggesting no effect of tRNS on inhibitory control in healthy subjects. However, we found that tRNS can acutely decreased 
reaction times at the Go trials as compared to sham suggesting an increased response execution after tRNS sessions. While no significant differences were observed between $1 \mathrm{~A} 2 \mathrm{~S}$ and $3 \mathrm{~A}$ groups, only the $3 \mathrm{~A}$ group differed from the $3 \mathrm{~S}$ group at 8-day follow-up. The absence of significant difference observed at T3 between participants who received at least one active session (3A and $1 \mathrm{~A} 2 \mathrm{~S}$ ) and $3 \mathrm{~S}$ were unexpected results that could be explained by a lack of power of the study. In line with this, one can also hypothesized that a significant difference could also be observed between $3 \mathrm{~A}$ and $1 \mathrm{~A} 2 \mathrm{~S}$ and between $1 \mathrm{~A} 2 \mathrm{~S}$ and $3 \mathrm{~S}$ at D8 with a larger sample size. Taken together, our results suggested that tRNS can acutely and sustainably improve performances in the Go/ No Go task by modulating the speed/accuracy trade-off in healthy controls.

The lack of effect of tRNS on accuracy in the Go/No Go task can be explained by several points. First, in our study, healthy subjects made very few false alarms and no omissions at baseline, raising the possibility that the Go/ No Go task was too easy (easily discriminable Go and NoGo stimuli and simple motor response) and that a floor effect contributed to the lack of significant effects of tRNS on Go/No-Go accuracy in healthy subjects without a default of inhibitory control. Second, this result is in accordance with numerous studies that reported no effect of tDCS on false alarms at the Go/No-Go task when targeting the DLPFC in healthy subjects $[3,9]$ and with the review and meta-analysis of Dedoncker et al. [10] showing that healthy subjects preferentially decrease reaction time than increase accuracy during low level cognitive tasks.

Regarding reaction times, we reported no significant difference between $3 \mathrm{~A}$ and $1 \mathrm{~A} 2 \mathrm{~S}$ throughout the study period and a significant longer lasting effect after $3 \mathrm{~A}$ as compared to $3 \mathrm{~S}$, suggesting that repeating tRNS sessions with a 30-minute interval enhance the duration but not the magnitude of the effects of tRNS on response 
execution. These findings are congruent with Monte-Silva et al.'s study [18] reporting that repeated tDCS sessions over the motor region induced prolonged and enhanced effects on motor cortex excitability with a short interval between sessions compared to a single session. The beneficial long-lasting effects after repeated sessions of stimulation were also in line with Bastani et al.'s study [2] showing that repeated tDCS sessions have long-lasting effects on corticospinal excitability and motor performance at D1 when the sessions are separated by 25 -min intervals as compared to when they are delivered with shorter intervals.

This study has several limits that should be acknowledged. The first limit is the small sample size of participants that led to undertake a rather underpowered statistical multi-comparison analysis given the between-subjects approach. Results of this pilot study should thus be taken with caution and further studies with larger sample size are needed to confirm present promising observations. A second limit of the study could be the level of impulsivity of participants at baseline that might have impact on performances at the Go/No Go task and then, in turn on tRNS effects on inhibitory control. To control for this bias, we measured impulsive behavior at baseline and reported no difference between groups using BIS10 scores. However, more recent scale such as the UPPS could help us to measure accurately impulsive behavior [24]. A third limit is that our study did not allow us to disentangle tRNS-induced effects on several cognitive functions such as planning, delay aversion or risk taking that are linked to DLPFC activity [5].

In summary, repeated tRNS sessions applied over the DLPFCs was not efficient to induce effects on inhibitory control in healthy subjects but can induce long lasting effects on speed of execution as compared to sham tRNS and a single session active 
session. Given the implication of inhibitory control in impulsive behaviors, repeated sessions of tRNS might show promise in patients with impulsive disorders. 


\section{Declaration of interest: none}

Funding: This study was supported by "conseil scientifique de la recherche" CSR CH Le Vinatier (CSRH01).

\section{References}

[1] Bari A, Robbins TW. Inhibition and impulsivity: behavioral and neural basis of response control. Prog Neurobiol 2013;108:44-79.

[2] Bastani A, Jaberzadeh S. Within-session repeated a-tDCS: the effects of repetition rate and inter-stimulus interval on corticospinal excitability and motor performance. Clin Neurophysiol 2014;125:1809-18.

[3] Beeli G, Casutt G, Baumgartner T, Jäncke L. Modulating presence and impulsiveness by external stimulation of the brain. Behav Brain Funct $2008 ; 4: 33$.

[4] Burton CZ, Vella L, Weller JA, Twamley EW. Differential effects of executive functioning on suicide attempts. J Neuropsychiatry Clin Neurosci 2011;23:1739.

[5] Brevet-Aeby C, Brunelin J, Iceta S, Padovan C, Poulet E. Prefrontal cortex and impulsivity: Interest of noninvasive brain stimulation. Neurosci Biobehav Rev $2016 ; 71: 112-34$.

[6] Boggio PS, Bermpohl F, Vergara AO, Muniz AL, Nahas FH, Leme PB, et al. Gono-go task performance improvement after anodal transcranial DC stimulation of the left dorsolateral prefrontal cortex in major depression. J Affect Disord 2007;101:91-8.

[7] Boggio PS, Liguori P, Sultani N, Rezende L, Fecteau S, Fregni F. Cumulative 
priming effects of cortical stimulation on smoking cue-induced craving. Neurosci Lett 2009;463:82-6.

[8] Chambers CD, Garavan H, Bellgrove MA. Insights into the neural basis of response inhibition from cognitive and clinical neuroscience. Neurosci Biobehav Rev 2009;33:631-46.

[9] Dambacher F, Schuhmann T, Lobbestael J, Arntz A, Brugman S, Sack AT. No effects of bilateral tDCS over inferior frontal gyrus on response inhibition and aggression. PloS One 2015;10:e0132170.

[10] Dedoncker J, Brunoni AR, Baeken C, Vanderhasselt MA. A systematic review and meta-analysis of the effects of transcranial direct current stimulation (tDCS) over the dorsolateral prefrontal cortex in healthy and neuropsychiatric samples: influence of stimulation parameters. Brain Stimul 2016;9:501-17.

[11] Fertonani A, Pirulli C, Miniussi C. Random noise stimulation improves neuroplasticity in perceptual learning. J Neurosci 2011;31:15416-23.

[12] Forster KI, Forster JC. DMDX: A Windows display program with millisecond accuracy. Behav Res Methods Instrum Comput 2003;35:116-24.

[13] Garavan H, Hester R, Murphy K, Fassbender C, Kelly C. Individual differences in the functional neuroanatomy of inhibitory control. Brain Res 2006;1105:13042.

[14] Ho KA, Taylor JL, Loo CK. Comparison of the effects of transcranial random noise stimulation and transcranial direct current stimulation on motor cortical excitability. J ECT 2015;31:67-72.

[15] Ho KA, Taylor JL, Chew T, Gálvez V, Alonzo A, Bai S, et al. The Effect of Transcranial Direct Current Stimulation (tDCS) Electrode Size and Current Intensity on Motor Cortical Excitability: Evidence From Single and Repeated 
Sessions. Brain Stimul 2016;9:1-7.

[16] Inukai Y, Saito K, Sasaki R, Tsuiki S, Miyaguchi S, Kojima S, et al. Comparison of three non-invasive transcranial electrical stimulation methods for increasing cortical excitability. Front Hum Neurosci 2016;10:668.

[17] Menon V, Adleman NE, White CD, Glover GH, Reiss AL. Error-related brain activation during a Go/NoGo response inhibition task. Hum Brain Mapp 2001;12:131-43.

[18] Monte-Silva K, Kuo MF, Hessenthaler S, Fresnoza S, Liebetanz D, Paulus W, et al. Induction of late LTP-like plasticity in the human motor cortex by repeated non-invasive brain stimulation. Brain Stimul 2013;6:424-32.

[19] Nieratschker V, Kiefer C, Giel K, Krüger R, Plewnia C. The COMT Val/Met polymorphism modulates effects of tDCS on response inhibition. Brain Stimul $2015 ; 8: 283-8$.

[20] Simmonds DJ, Pekar JJ, Mostofsky SH. Meta-analysis of Go/No-go tasks demonstrating that fMRI activation associated with response inhibition is taskdependent. Neuropsychologia 2008;15;46:224-32.

[21] Soltaninejad Z, Nejati V, Ekhtiari H. Effect of anodal and cathodal transcranial direct current stimulation on DLPFC on modulation of inhibitory control in ADHD. J Atten Disord 2015; in press.

[22] Talsma LJ, Kroese HA, Slagter HA. Boosting Cognition: Effects of MultipleSession Transcranial Direct Current Stimulation on Working Memory. J Cogn Neurosci 2017;29:755-68.

[23] Terney D, Chaieb L, Moliadze V, Antal A, Paulus W. Increasing human brain excitability by transcranial high-frequency random noise stimulation. J Neurosci 2008;28:14147-55. 
[24] Whiteside SP, Lynam DR. The five factor model and impulsivity: Using a structural model of personality to understand impulsivity. Pers Individ Diff 2001;30:669-89.

[25] Zappasodi F, Musumeci G, Navarra R, Di Lazzaro V, Caulo M, Uncini A. Safety and effects on motor cortex excitability of five cathodal transcranial direct current stimulation sessions in 25 hours. Neurophysiol Clin 2017;48:77-87. 
Table 1: Sociodemographic and clinical characteristics of the 33 healthy participants included in the study.

\begin{tabular}{lllll} 
& $3 \mathrm{~A}$ & $1 \mathrm{~A} 2 \mathrm{~S}$ & $3 \mathrm{~S}$ & $\mathrm{p}$ \\
\hline $\mathrm{N}(\mathrm{W} / \mathrm{M})$ & $12(6 / 6)$ & $10(4 / 6)$ & $11(6 / 5)$ & $0.83^{\mathrm{a}}$ \\
Age (years) & $23.2(3.5)$ & $25.7(4.9)$ & $25.5(4.5)$ & $0.30^{\mathrm{b}}$ \\
Educational level (years) & $15.0(2.8)$ & $16.3(2.8)$ & $16.5(2.7)$ & $0.36^{\mathrm{b}}$ \\
Impulsivity (BIS10 Barratt Scale scores) & $65.3(6.5)$ & $70.9(6.8)$ & $65.3(7.6)$ & $0.13^{\mathrm{b}}$
\end{tabular}

The results are given as the mean (+standard deviation). 3A group: participants received 3 active sessions; 1A2S: participants received 1 active and 2 sham sessions; 3S: participants received 3 sham sessions. ${ }^{a}$ Fisher Exact test; ${ }^{b}$ One Way ANOVA. 
Figure 1. A) Experimental design of the study. 33 participants were randomly assigned to receive either three 20-min sessions of active transcranial random noise stimulation (tRNS, 3A, n= 12), 1 active and 2 sham sessions of tRNS (1A2S, n=10) or 3 sham tRNS sessions $(3 \mathrm{~S}, \mathrm{n}=11)$. Sessions were delivered in the same day separated by 30 minutes. Accuracy and mean reaction times at the Go/No-Go task were assessed 5 times, at baseline (T0), after one and three tRNS sessions (T1, T3), one day (D1) and 8 days (D8) after tRNS; B) Example of the stimulus presentation sequence during the verbal Go/No-Go task. In this example, the letter ' $\mathrm{B}$ ' is a Go stimulus that require a quick key-press response, whereas the letter ' $E$ ' is a No-Go stimulus that require the response to be withheld; C) Effects of transcranial random noise stimulation (tRNS) applied over the dorsolateral prefrontal cortex on reaction times in the Go trials in healthy subjects. At each time point, changes in mean reaction times are expressed as a ratio to baseline (T0) (+/-standard error of the mean). ns: not significant; $*$ : significant $\mathrm{p}<0.05$ 
A)

Active tRNS session

B)

Sham tRNS session
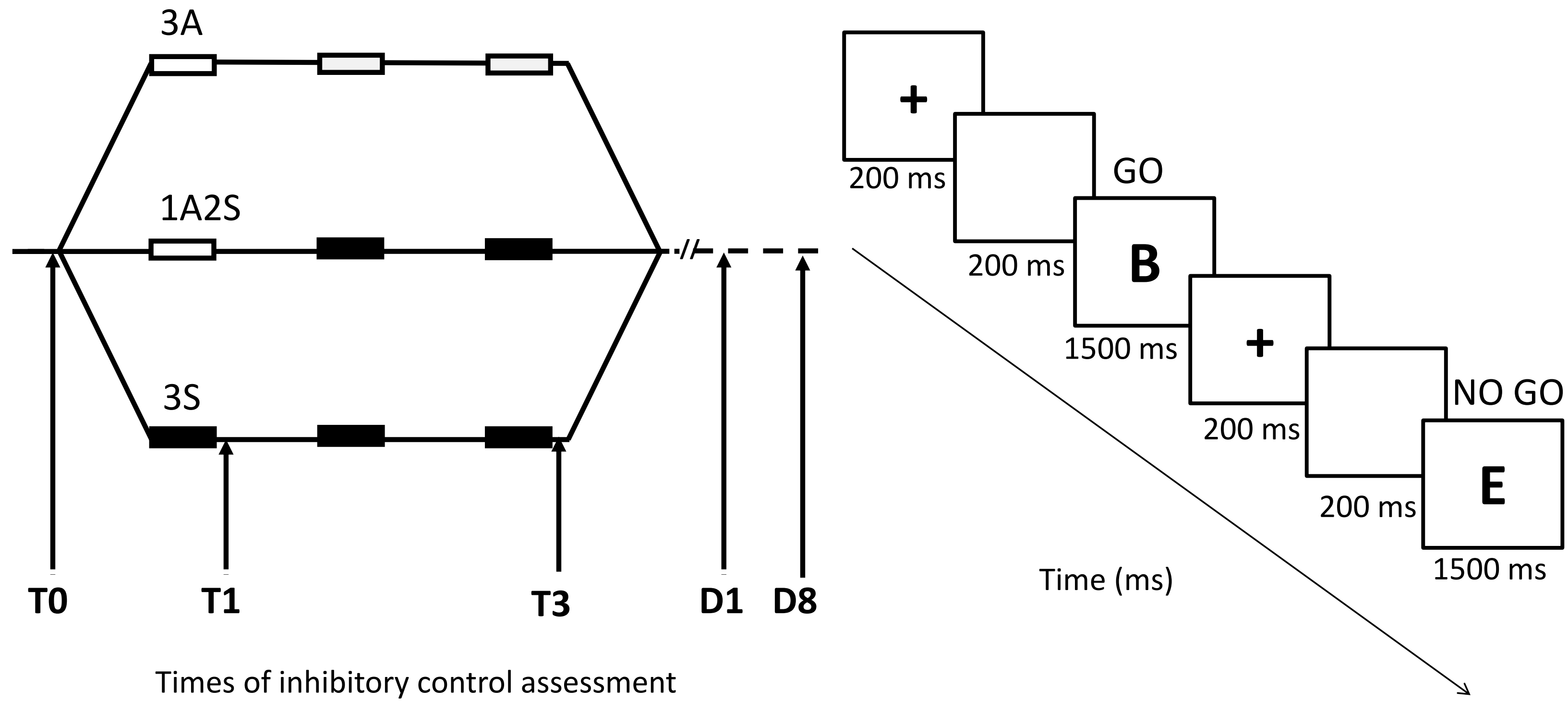

C)

Times of inhibitory control assessment

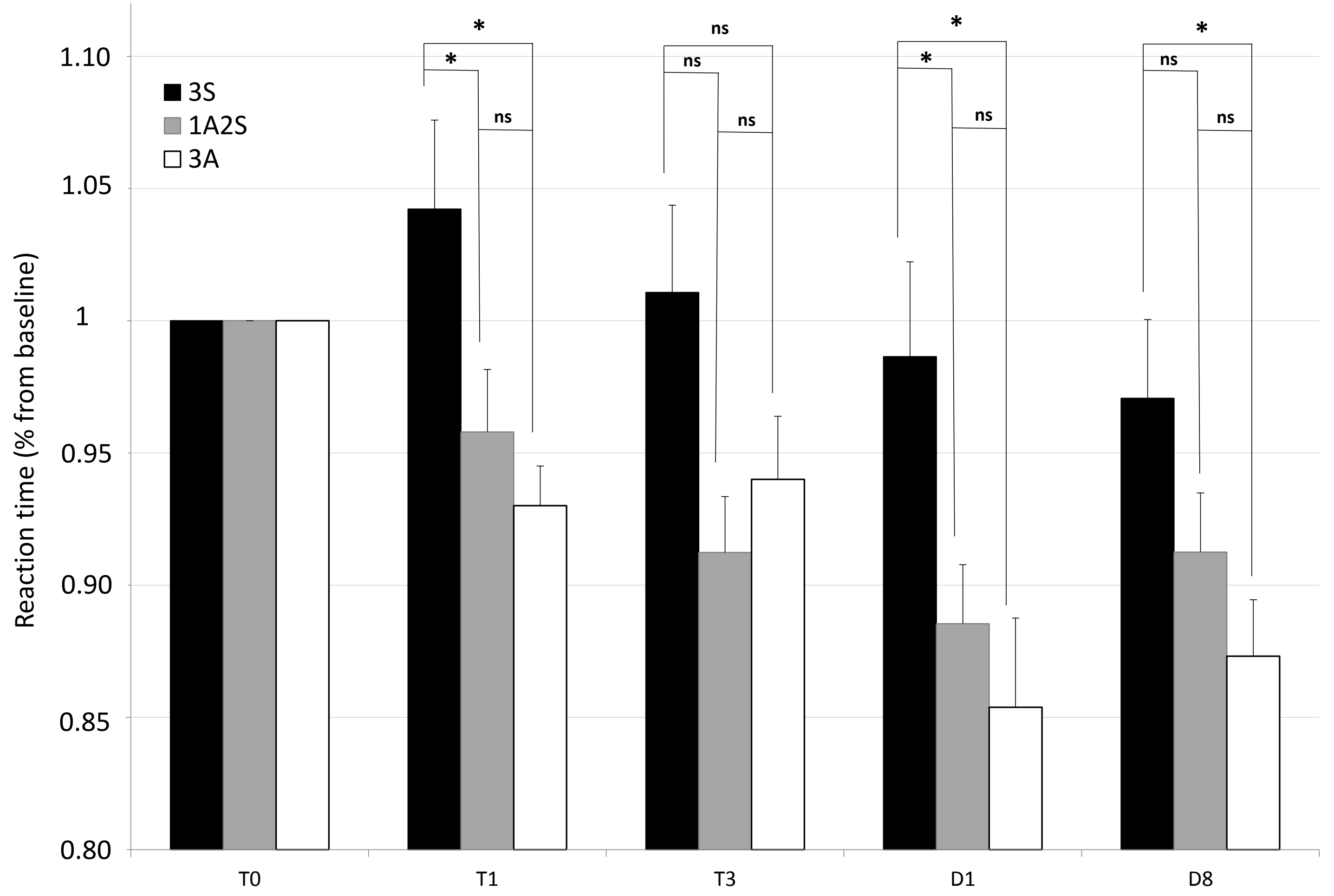

